\title{
Patchy screening of the cosmic microwave background by inhomogeneous reionization
}

\author{
Vera Gluscevic, ${ }^{1}$ Marc Kamionkowski, ${ }^{2}$ and Duncan Hanson ${ }^{3}$ \\ ${ }^{1}$ California Institute of Technology, Mail Code 350-17, Pasadena, California 91125, USA \\ ${ }^{2}$ Department of Physics and Astronomy, Johns Hopkins University, Baltimore, Maryland 21218, USA \\ ${ }^{3}$ Department of Physics, McGill University, Montreal, Quebec H3A 2T8, Canada
}

(Received 1 November 2012; published 27 February 2013)

\begin{abstract}
We derive a constraint on patchy screening of the cosmic microwave background from inhomogeneous reionization using off-diagonal $T B$ and $T T$ correlations in WMAP-7 temperature/polarization data. We interpret this as a constraint on the rms optical-depth fluctuation $\Delta \tau$ as a function of a coherence multipole $L_{C}$. We relate these parameters to a comoving coherence scale, of bubble size $R_{C}$, in a phenomenological model where reionization is instantaneous but occurs on a crinkly surface, and also to the bubble size in a model of "Swiss cheese" reionization where bubbles of fixed size are spread over some range of redshifts. The current WMAP data are still too weak, by several orders of magnitude, to constrain reasonable models, but forthcoming Planck and future EPIC data should begin to approach interesting regimes of parameter space. We also present constraints on the parameter space imposed by the recent results from the EDGES experiment.
\end{abstract}

DOI: 10.1103/PhysRevD.87.047303

PACS numbers: $98.70 . V_{c}$

\section{INTRODUCTION}

Simple estimates have long shown that radiation from the first star-forming galaxies in cold-dark-matter models should reionize the intergalactic medium at redshifts $z \sim 10$ [1], but the details of this Epoch of Reionization are still unclear. Quasar observations suggest that the tail end of reionization occurred at a redshift $z \gtrsim 6$ [2], but the implications of these measurements are difficult to interpret precisely [3]. A constraint $\tau=0.074 \pm 0.034$ to the optical depth to rescattering of cosmic-microwavebackground $(\mathrm{CMB})$ photons suggests a reionization redshift $z=10.6 \pm 1.4[4,5]$ if reionization occurred everywhere suddenly. However, the current paradigm is that reionization was highly inhomogeneous [6]: regions of ionized gas, seeded by the first light sources, grew and eventually coalesced, filling the entire volume of the intergalactic medium. If so, then reionization may have occurred over some redshift range. Recent searches for the redshifted $21-\mathrm{cm}$ signal from neutral hydrogen $[7,8]$ now suggest that reionization occurred over a redshift range $\Delta z \geq 0.06$ [9]. The South Pole Telescope collaboration has now bounded that redshift range from above to be $\Delta z \lesssim 7.9$ [10], by searching for a fluctuating kinetic Sunyaev-Zeldovich (kSZ) signal [11], under assumptions that the mechanism of reionization accords with prevailing theoretical models.

Here we study the effects of patchy screening on the CMB [12]. Thomson scattering of CMB photons during the Epoch of Reionization damps small-scale CMB fluctuations by a factor $e^{-\tau(\hat{\mathbf{n}})}$, where $\tau(\hat{\mathbf{n}})$ is the optical depth in direction $\hat{\mathbf{n}}$ on the sky. Patchy screening gives rise to a direction-dependent optical depth $\tau(\hat{\mathbf{n}})$. This then produces a $\mathrm{B}$-mode polarization that is correlated in a characteristic way with the temperature and with the E-mode polarization $[12,13]$, and it also modulates the power in the temperature map. Here we interpret prior null searches for a modulation of CMB power [14] in terms of an upper limit to optical-depth fluctuations, and we apply a minimum-variance estimator [12] for $\tau$ fluctuations to the WMAP-7 temperature and polarization maps [15] to search for patchy screening by measuring the off-diagonal $T B$ correlations. We derive an upper limit to all multipoles of the power spectrum $C_{L}^{\tau \tau}$ up to $L=512$. We then discuss implications of these constraints for a simple phenomenological reionization model whose parameters might serve as figures of merit for future experiments. We revisit predictions for future experiments and discuss constraints on the parameter space imposed by the recent results from the EDGES [9] experiment.

\section{FORMALISM}

Patchy screening suppresses primary anisotropies (marked with tilde), so the observed temperature fluctuation and polarization are, respectively,

$$
\begin{aligned}
\Delta T(\hat{\mathbf{n}}) & =e^{-\tau(\hat{\mathbf{n}})} \widetilde{\Delta T}(\hat{\mathbf{n}}), \\
p(\hat{\mathbf{n}}) & \equiv Q(\hat{\mathbf{n}})+i U(\hat{\mathbf{n}})=e^{-\tau(\hat{\mathbf{n}})} \tilde{p}(\hat{\mathbf{n}}),
\end{aligned}
$$

where $Q$ and $U$ are the usual Stokes parameters. All temperature and polarization correlations in the CMB can, in principle, be used to reconstruct the map of $\tau(\hat{\mathbf{n}})$. The $E B$ estimator will ultimately provide the best sensitivity to patchy screening $[13,16]$, once low-noise polarization measurements are available with future CMB exeriments. With WMAP and Planck [17], however, the best sensitivity is achieved with the $T T$ correlation, which we discuss below. Here, we derive a constraint to patchy screening from the $T B$ correlation, as a proof of principle. The estimator for the optical-depth fluctuation is $[13,18]$, 


$$
\begin{aligned}
\hat{\tau}_{L M}= & -i N_{L} \int d \hat{\mathbf{n}} Y_{L M}(\hat{\mathbf{n}}) \\
& \times\left[\sum_{l m l^{\prime} m^{\prime}} \bar{B}_{l m 2}^{*} Y_{l m}(\hat{\mathbf{n}}) \tilde{C}_{l^{\prime}}^{T E} \bar{T}_{l^{\prime} m^{\prime} 2} Y_{l^{\prime} m^{\prime}}^{*}(\hat{\mathbf{n}})+\mathrm{cc}\right],
\end{aligned}
$$

where $Y_{l m}$ and ${ }_{2} Y_{l m}$ are spherical harmonics and spinweighted spherical harmonics, respectively, and the sum is only over $l+l^{\prime}+L=$ odd. The unbarred $B_{l m}$ and $T_{l m}$ are the observed temperature and polarization multipoles recovered from the maps and corrected for the combined instrumental beam and pixelization transfer function $W_{l}$; bars represent the inverse-variance-filtered multipoles, $\bar{B}_{l m} \equiv B_{l m} / C_{l}^{B B}$ and $\bar{T}_{l m} \equiv T_{l m} / C_{l}^{T T}$, where the $T T$ and $B B$ power spectra are analytic estimates of the total (signal plus noise) power spectrum $C_{l}^{X X} \equiv \tilde{C}_{l}^{X X}+C_{l}^{X X \text {,noise }} W_{l}^{-2}$ in a given frequency band, for $X X \in\{T T, B B\}$. The normalization $N_{L}$ can be calculated either analytically or using Monte Carlo simulations. The estimator is equivalent to the real part of the cosmic-birefringence estimator in Refs. $[16,18]$, the only difference being the parity condition. Reference [18] demonstrated that the full-sky formalism with the full-sky inverse-variance-filtered procedure described above is justified in spite of the sky cuts introduced by masking the Galaxy.

The $T B$ correlations sought by this estimator can in principle also be generated by rescattering of CMB photons and by the kSZ effect from rescattering. However, Ref. [13] showed that the estimator is relatively insensitive to the kSZ effect, and also that only the large-scale $(l \lesssim 40)$ temperature fluctuations are sensitive to the former mechanism. In order to avoid large-scale contamination from pixel-pixel noise correlations in WMAP, we discard $T_{l m}$ and $E_{l m}$ multipoles below $l=100$ from our analysis anyway, so we effectively probe only patchy screening.

The estimator for the corresponding power spectrum of fluctuations of $\tau$ is

$$
C_{L}^{\hat{\tau} \hat{\tau}} \equiv\left[f_{\text {sky }}(2 L+1)\right]^{-1} \sum_{M} \hat{\tau}_{L M} \hat{\tau}_{L M}^{*},
$$

where $f_{\text {sky }}$ represents the fraction of the sky admitted by the analysis mask, correcting for the fact that the full-sky analysis is applied to the maps where a portion of the pixel values (mostly around the Galactic plane) was set to zero. When evaluated for the fixed cosmology of Ref. [4] and for the noise levels appropriate for the experiment in consideration, this four-point correlation provides a biased estimate of $C_{L}^{\tau \tau}$, where the bias mostly arises from the inhomogeneous pixel noise and the sky cuts. However, if this trispectrum is estimated by cross correlating the $\hat{\tau}_{L M}$ signal estimated from one frequency band with the same signal estimated from another frequency band, the largest contribution to its bias vanishes, because the instrumental noise is uncorrelated in different frequency bands. The leftover bias can be evaluated and subtracted by running a suite of null-hypothesis (no patchy signal) Monte Carlo simulations. We also use the null simulations to recover the statistical uncertainty for each measurement following the procedure described in Ref. [18].

\section{RESULTS FROM TB ESTIMATOR}

We only show results for the cleanest band-crosscorrelation [WW][VV] where the estimate of $\tau_{L M}$ recovered from the $\mathrm{W}$ band is cross correlated with the estimate from the $\mathrm{V}$ band. Prior to the analysis, we mask out the Galaxy and the known point sources using the fiducial seven-year analysis masks available at the LAMBDA website [15] (where for the combined mask $f_{\text {sky }} \simeq 68 \%$ ). After subtracting the bias, we recover a debiased estimate $\hat{C}_{L}^{\tau \tau}$ of the power spectrum at each multipole up to $L=512$; Fig. 1 shows the binned measurements with estimated uncertainties. At all multipoles, we recover consistency with zero within the $3 \sigma$ confidence level.

\section{TESTS OF SYSTEMATICS}

Our simulations do not include polarized point sources nor foreground residuals. In order to test their impact on our estimates of the power spectrum and associated statistical uncertainty, we perform the tests described in Ref. [18]. The results of these tests are shown in Figs. 2 and 3, which demonstrate that the foregrounds and point

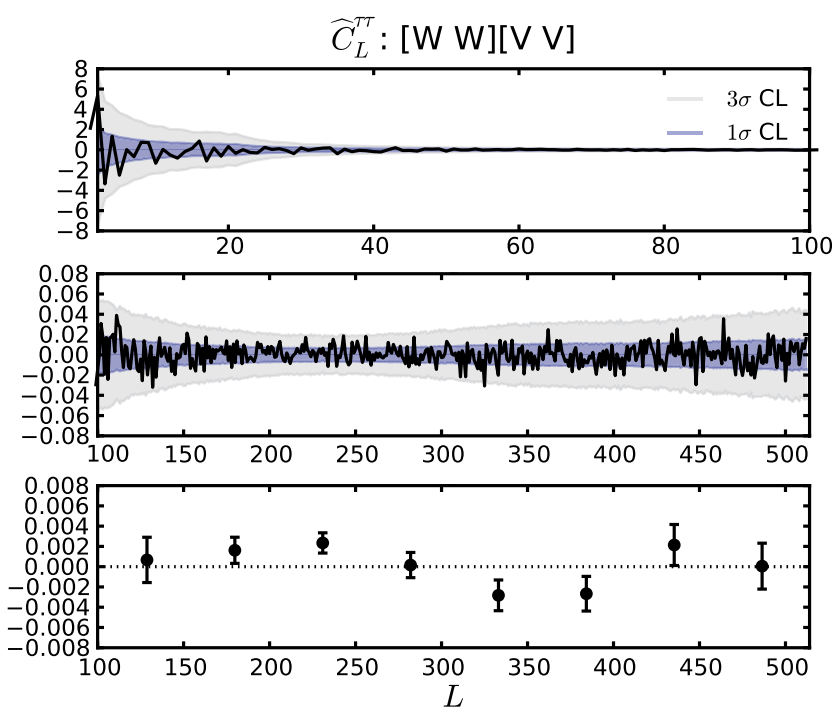

FIG. 1 (color online). The measurement of the power spectrum of fluctuations of the optical depth $\tau$ from $T B$ correlation with corresponding $1 \sigma$ and $3 \sigma$ confidence-level intervals for all multipoles up to the resolution limit of WMAP-7 is shown in the top two panels. A binned version with associated statistical uncertainty is shown in the bottom panel. The first two bins are $-0.0085 \pm 0.1264$ at $L=26$, and $0.0029 \pm 0.0056$ at $L=77$; they are omitted for the sake of clearer presentation. The measurements are consistent with zero at all multipoles. 


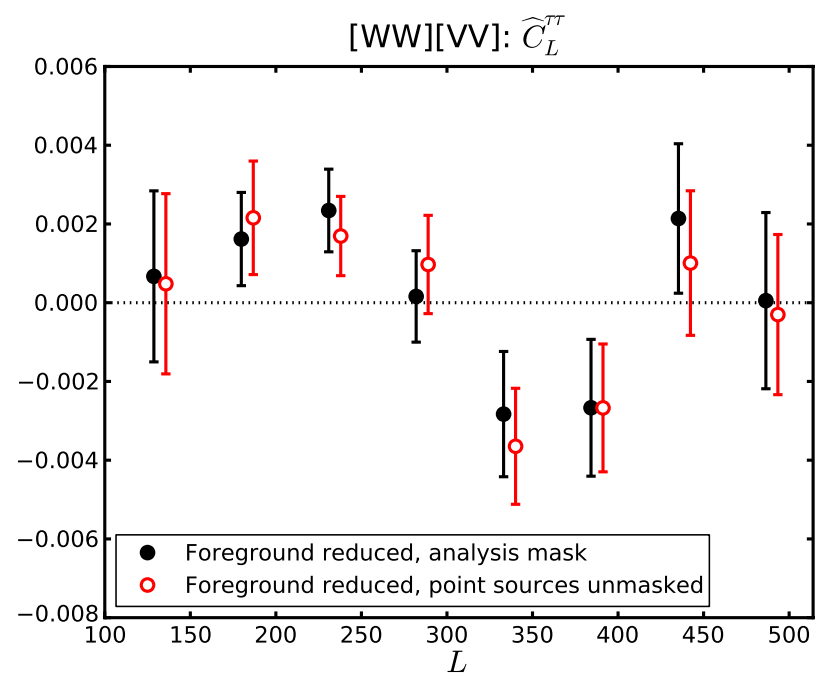

FIG. 2 (color online). Measurement of $\hat{C}_{L}^{\tau \tau}$ from $T B$ correlation in WMAP-7 data. Results shown in black (filled circles) are obtained by using the analysis mask that covers all the point sources brighter than $\sim 1 \mathrm{Jy}$, while the results in red (empty circles) are obtained after unmasking all the point sources. In spite of the large difference in the source contamination, the two results differ by much less than the statistical uncertainty, and no overall bias is observed.

sources do not significantly affect the results reported in Fig. 1.

\section{INTERPRETATION}

To understand the implications of these measurements, we consider a simple parametrization of inhomogeneous reionization in which optical-depth fluctuations are described by white noise smoothed on angular scales $\theta_{C} \equiv \pi / L_{C}$, or a power spectrum

$$
C_{L}^{\tau \tau}=\left(4 \pi / L_{C}^{2}\right)(\Delta \tau)^{2} e^{-L^{2} / L_{C}^{2}},
$$

shown in Fig. 4 for several values of $L_{C}$. We constrain the parameters $\Delta \tau$ and $L_{C}$ using the minimum-variance estimate [19] for the amplitude,

$$
\widehat{(\Delta \tau}^{2}=\left(\sigma\left[(\Delta \tau)^{2}\right]\right)^{2} \sum_{L} C_{L}^{\tau \tau, \text { fiducial }} \hat{C}_{L}^{\tau \tau} / \operatorname{var}\left(\hat{C}_{L}^{\tau \tau}\right),
$$

where

$$
\left(\sigma\left[(\Delta \tau)^{2}\right]\right)^{-2}=\sum_{L}\left(C_{L}^{\tau \tau, \text { fiducial }}\right)^{2} / \operatorname{var}\left(\hat{C}_{L}^{\tau \tau}\right)
$$

is roughly the inverse variance with which $(\Delta \tau)^{2}$ can be measured, $\operatorname{var}\left(\hat{C}_{L}^{\tau \tau}\right)$ is the variance of the power spectrum estimated from a suite of simulations with no patchy screening, and $\hat{C}_{L}^{\tau \tau}$ are the unbinned measurements from WMAP-7 maps. Since the results are consistent with no signal, the variance provides a constraint on $(\Delta \tau)^{2}$, which we show as a function of the model parameter $L_{C}$ in Fig. 5. We also show in Fig. 5 the upper limit to $(\Delta \tau)^{2}$ inferred

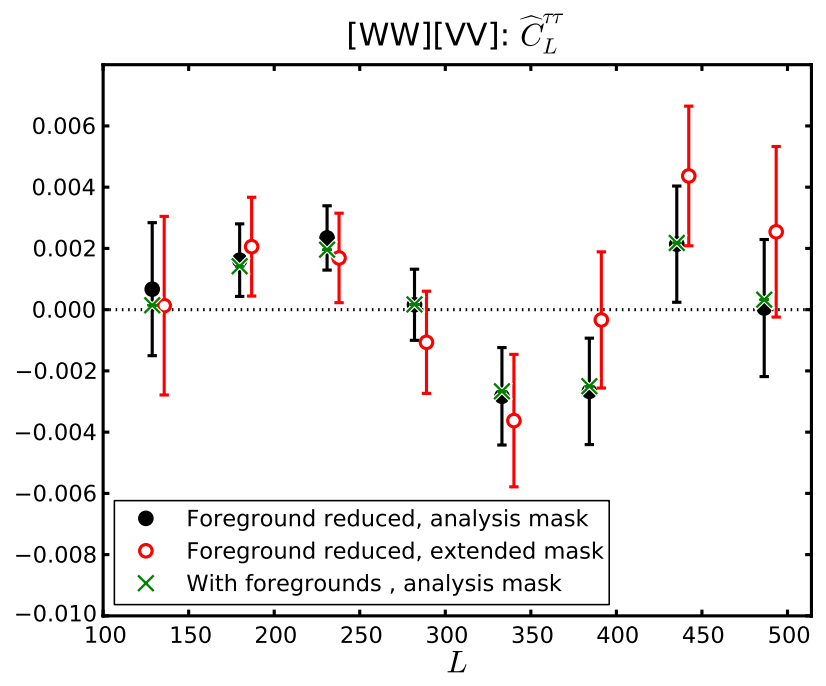

FIG. 3 (color online). Measurement of $\hat{C}_{L}^{\tau \tau}$ from $T B$ correlation in WMAP-7 data. Black filled circles represent the measurements obtained from the foreground-reduced maps after applying the fiducial analysis mask (the fiducial result of Fig. 1). The rest of the data points correspond to the two test cases: the green x's are obtained from the maps prior to foreground subtraction, but using the fiducial mask, while the red empty circles are measurements obtained from foregroundreduced maps after applying an extended mask. No overall bias is observed in the two cases, and all three results are consistent, within the estimated statistical uncertainty.

from upper limits to the power of $T T$ modulation [14]. Given that the mean optical depth is known to be $\tau \sim 0.1$, it is clear that our bounds $\Delta \tau \lesssim 1$, from $T B$, are far from constraining, and that $\Delta \tau \lesssim 0.01-0.1$, from $T T$, are at best marginally constraining.

Equation (4) describes what happens if every point in the Universe goes suddenly from neutral to ionized, but with a reionization surface that is crinkled on a comoving scale of

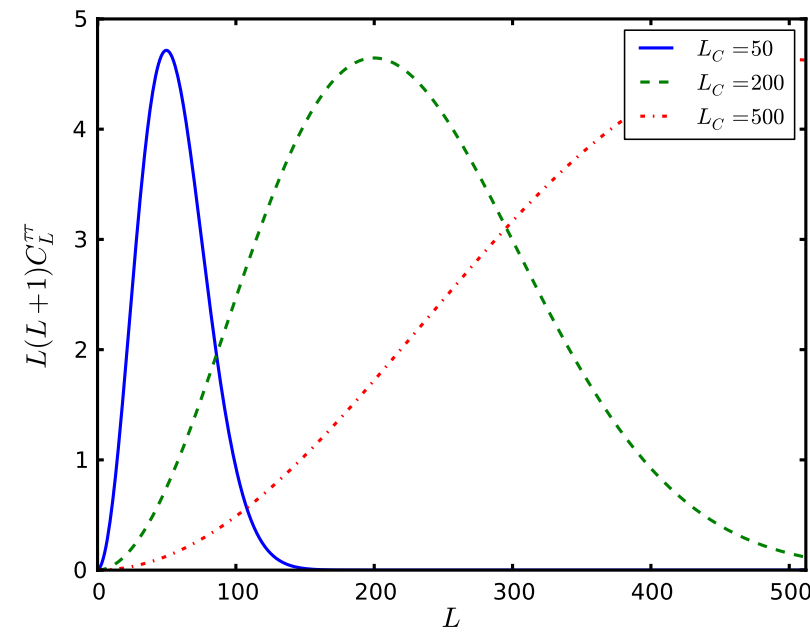

FIG. 4 (color online). Family of simple patchy-reionization models given by Eq. (4) for $\Delta \tau=1$ and different values of $L_{C}$. 


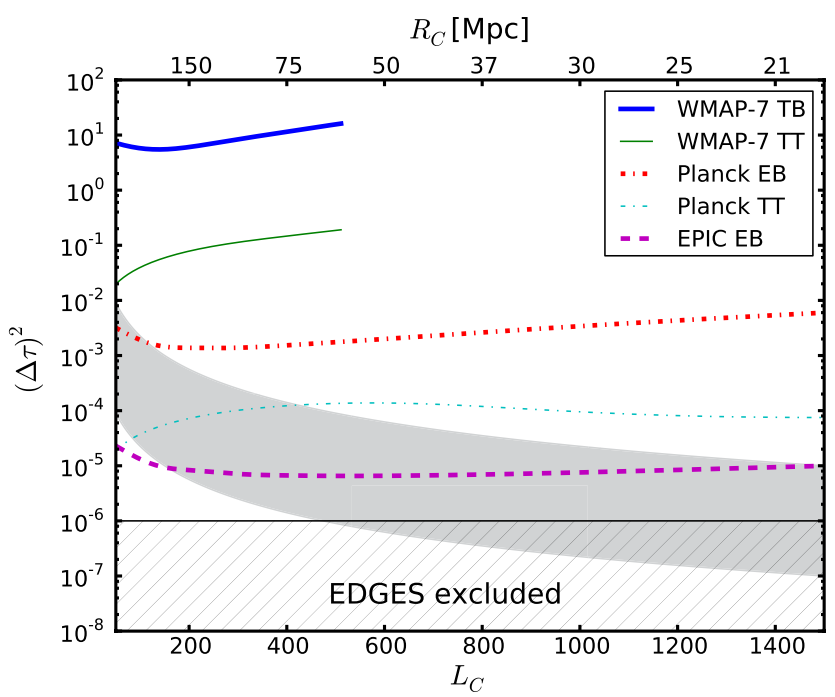

FIG. 5 (color online). Shown are the $1 \sigma$ confidence-level upper limits from WMAP-7 $T T$ and $T B$ correlations on the amplitude $(\Delta \tau)^{2}$ of the patchy-screening model given in Eq. (4) as a function of the coherence-scale parameter $L_{C}$. Also shown are sensitivity forecasts for experiments with map noise of $27 \mu \mathrm{Karcmin}$ and $1 \mu \mathrm{Karcmin}$, and beam width of $7^{\prime}$ and $5^{\prime}$, corresponding to Planck and EPIC-like mission, respectively. The values expected for a reionization surface that is crinkled on scales $R_{C}$ are indicated by the gray band. Also shown is the portion of this parameter space excluded by EDGES [9].

$R_{C} \simeq 200 \mathrm{Mpc}\left(L_{C} / 150\right)^{-1}$. This smoothing scale, or bubble size, corresponds at a reionization redshift $z_{r} \sim 10$ to a redshift interval $\Delta z \sim R_{C} z_{r}^{1 / 2} \Omega_{m}^{1 / 2} H_{0} / c$. Since the optical depth scales with the reionization redshift as $\tau \propto z^{3 / 2}$, we find that a bubble size $R_{C}$ induces an optical-depth fluctuation $\Delta \tau \simeq 0.01\left(R_{C} / 200 \mathrm{Mpc}\right)$. There is thus a rough scaling, $(\Delta \tau) \sim 0.01\left(L_{C} / 150\right)^{-1}$, between the opticaldepth-fluctuation amplitude and the correlation multipole $L_{C}$ for the crinkly-surface model represented by a thick band (to indicate roughly the theory uncertainty) in Fig. 5. Figure 5 also shows the expectations $[13,16]$ for the sensitivities of Planck and EPIC [20]. Also shown is a constraint for this crinkly-surface model from the EDGES constraint, $\Delta z>0.06$ (at 95\% confidence) [9], from the all-sky redshifted 21-cm spectrum.

A wider range of reionization scenarios can be described by a "Swiss cheese" model in which bubbles of size $R_{C}$ are spread over a larger redshift range [11], so that each line of sight crosses, on average, $N$ bubbles. The rms opticaldepth fluctuation in Eq. (5) would, for fixed $R_{C}$, then be reduced by a factor $N^{1 / 2}$, relative to the crinkly-surface model. Thus, both the gray shaded area, and the "EDGES excluded" regions in Fig. 5 would be reduced by $N^{1 / 2}$. Note that kSZ fluctuations should increase in sensitivity as $N$ increases $[10,11]$ to complement the reduced sensitivity of patchy screening in this limit.

\section{CONCLUSIONS}

The directional dependence of the optical depth $\tau(\hat{\mathbf{n}})$ encodes information about the morphology of the ionized regions during the Epoch of Reionization. Here we have used WMAP-7 temperature and polarization data to derive a bound on the individual multipoles of the optical-depth power spectrum up to $L=512$, or bubble sizes larger than $\sim 60 \mathrm{Mpc}$ comoving. We then interpreted these null results in terms of a bound on a rms optical-depth fluctuation $\Delta \tau$ in a model of white-noise fluctuations with coherence angle $\theta_{C}$. While the bound derived proves to be too weak to constrain realistic models, and probes bubble sizes larger than those $\left(R_{C} \lesssim 10 \mathrm{Mpc}\right)$ favored in current reionization models, our result provides a proof of principle that such analyses can be carried out with future data. We then note that data from the forthcoming Planck satellite and from a subsequent post-Planck project should approach the realistic parameter space. Before such optical-depth-fluctuation searches are carried out in the future, though, several issues will need to be understood. For example, the estimator in Eq. (3) has the same parity as that for the lensing potential [16,21], and further modeling of the $\Delta \tau$ and lensing signals, and/or delensing of the $\mathrm{CMB}$, will be necessary for a $\mathrm{CMB}$ detection in the optical-depth fluctuation with Planck [17] or future-generation experiments.

\section{ACKNOWLEDGMENTS}

The authors thank Gil Holder for useful discussions. This work was supported by the Simons Foundation at Caltech, Grant No. DoESC-0008108 and NASA Grant No. NNX12AE86G at Caltech, and a CITA National Fellowship at McGill. Some of the results in this paper have been derived using HEALPix [22].
[1] M. Kamionkowski, D. N. Spergel, and N. Sugiyama, Astrophys. J. 426, L57 (1994); M. Fukugita and T. Kawasaki, Mon. Not. R. Astron. Soc. 269, 563 (1994); P. R. Shapiro, M. L. Giroux, and A. Babul, Astrophys. J. 427, 25 (1994); M. Tegmark, J. Silk, and A. Blanchard, Astrophys. J. 420, 484 (1994).
[2] R. H. Becker et al. (SDSS Collaboration), Astron. J. 122, 2850 (2001); X. Fan, M. A. Strauss, R. H. Becker, R. L. White, J.E. Gunn, G. R. Knapp, G. T. Richards, D.P. Schneider, J. Brinkmann, and M. Fukugita, Astron. J. 132, 117 (2006).

[3] S. P. Oh and S. R. Furlanetto, Astrophys. J. 620, L9 (2005). 
[4] E. Komatsu et al. (WMAP Collaboration), Astrophys. J. Suppl. Ser. 192, 18 (2011).

[5] D. Larson et al. (WMAP Collaboration), Astrophys. J. Suppl. Ser. 192, 16 (2011).

[6] R. Barkana and A. Loeb, Phys. Rep. 349, 125 (2001).

[7] S. Furlanetto, S. P. Oh, and F. Briggs, Phys. Rep. 433, 181 (2006).

[8] J. R. Pritchard and A. Loeb, Rep. Prog. Phys. 75, 086901 (2012).

[9] J. D. Bowman and A. E. E. Rogers, Nature (London) 468, 796 (2010).

[10] O. Zahn et al. (SPT Collaboration), Astrophys. J. 756, 65 (2012).

[11] A. Gruzinov and W. Hu, Astrophys. J. 508, 435 (1998); L. Knox, R. Scoccimarro, and S. Dodelson, Phys. Rev. Lett. 81, 2004 (1998).

[12] C. Dvorkin, W. Hu, and K. M. Smith, Phys. Rev. D 79, 107302 (2009).
[13] C. Dvorkin and K. M. Smith, Phys. Rev. D 79, 043003 (2009).

[14] D. Hanson and A. Lewis, Phys. Rev. D 80, 063004 (2009).

[15] http://lambda.gsfc.nasa.gov/.

[16] V. Gluscevic, M. Kamionkowski, and A. Cooray, Phys. Rev. D 80, 023510 (2009).

[17] http://www.rssd.esa.int/PLANCK.

[18] V. Gluscevic, D. Hanson, M. Kamionkowski, and C. M. Hirata, Phys. Rev. D 86, 103529 (2012).

[19] V. Gluscevic and M. Kamionkowski, Phys. Rev. D 81, 123529 (2010).

[20] D. Baumann et al. (CMBPol Study Team Collaboration), AIP Conf. Proc. 1141, 3 (2009).

[21] C. M. Hirata and U. Seljak, Phys. Rev. D 68, 083002 (2003).

[22] K. M. Gorski, E. Hivon, A. J. Banday, B. D. Wandelt, F. K. Hansen, M. Reinecke, and M. Bartelmann, Astrophys. J. 622, 759 (2005); http://healpix.jpl.nasa.gov. 